\title{
Treatment of a patient with lumbosacral and pelvic fractures due to a blast injury without surgical stabilization
}

\section{- a case report}

\author{
Ioannis Spanos, Anastasios Venetikidis, Panagiotis Zogopoulos*, Georgios Vretakos, Dimitrios Rologis \\ Department of Neurosurgery, Metropolitan Hospital, Athens, Greece
}

\section{Introduction}

Blast injury to the spine and pelvis is part of a multisystem trauma, resulting from direct or indirect exposure to an explosion, encountered in war zones and in urban terrorist attacks $[1,2]$. They are high energy injuries associated with severe disabilities and increased mortality. We present the case of a young male patient with spinal and pelvic fractures due to a blast injury, who was treated without surgical stabilization because his contaminated wounds were an absolute contraindication for such a therapeutic approach.

\section{Case report}

A 24-year-old male was transferred to our hospital from another hospital abroad after having sustained a blast injury to the spine and pelvis. On admission he was fully alert and neurological examination revealed lower extremities hypoesthesia of S1 and S2 roots distribution (but no muscle weakness), saddle anesthesia and urine/bladder dysfunction. There was an incompletely healed trauma of the sacral region that presented cerebrospinal fluid leak, pus outflow and skin laceration. A lumbar drain had been inserted at L1 level (above the level of injury) at the previous hospital. Radiological examination with computerized tomography scans of the thoracic, lumbar and sacral spine, as well as pelvis, revealed fractures of the L5 and S1 vertebrae, dislocated fractures of the sacrum (S3,S4 and S5 segments) and bilateral sacroiliac joint fractures (Figures 1 and 2).
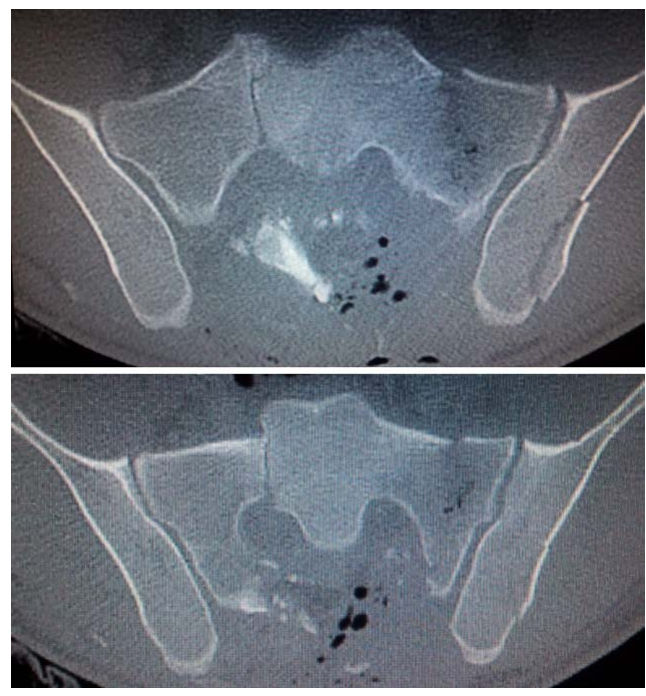

Figure 1. Admission axial CT scan images of the sacroiliac region.
The patient underwent surgical debridement of the sacral trauma, where pus and wood fragments were found and removed and dura was closed utilizing tissue glue (Figure 3). Due to the highly contaminated tissue environment a surgical stabilization of the lumbosacral spine and pelvis with screws and rods was contraindicated. Therefore, a new lumbar drain was inserted in order to facilitate dura closure and intensive antibiotic treatment was initiated. In order to accelerate wound healing, as well as augment bone union, hyperbaric oxygen therapy was also implemented.

After three months of treatment, along with intensive physiotherapy, follow-up CT scan of the lumbosacral spine revealed a satisfactory bone fusion without evidence of instability, thus without the necessity of surgical stabilization (Figures 4 and 5). The patient was mobilized with a lumbosacral support belt and foley catheter was removed, since control of bladder (and bowel) function was partially restored. He was finally discharged after four months of hospitalization, having good muscle strength and sensation of the lower extremities and adequate bladder and bowel control.

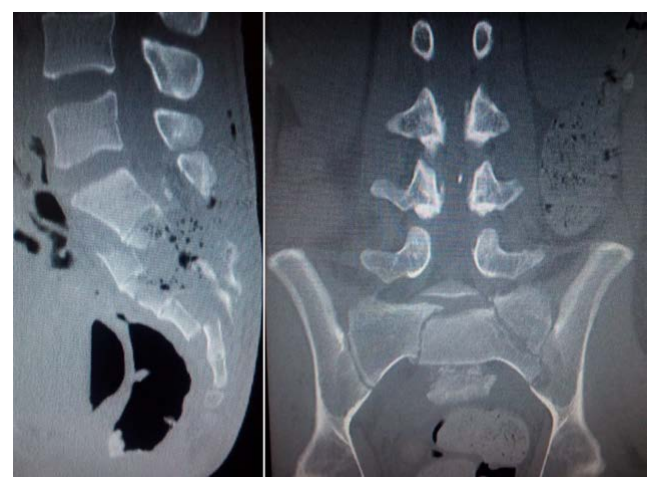

Figure 2. Admission sagittal and coronal CT scan images of the lumbosacral region.

Correspondence to: Panagiotis Zogopoulos, Department of Neurosurgery, Metropolitan Hospital, 69 Vosporou str., Athens, Greece, P.O. Box: 10444, Tel: +306976053555, E-mail: p.zogopoulos@yahoo.com

Key words: blast injury, lumbosacral fracture, pelvic fracture, conservative treatment, hyperbaric oxygen

Received: January 27, 2016; Accepted: February 20, 2016; Published: February 24, 2016 


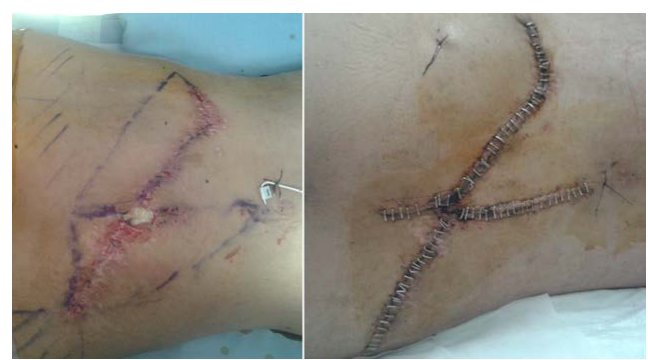

Figure 3. Admission photo of the contaminated back wound and photo after surgical debridement.

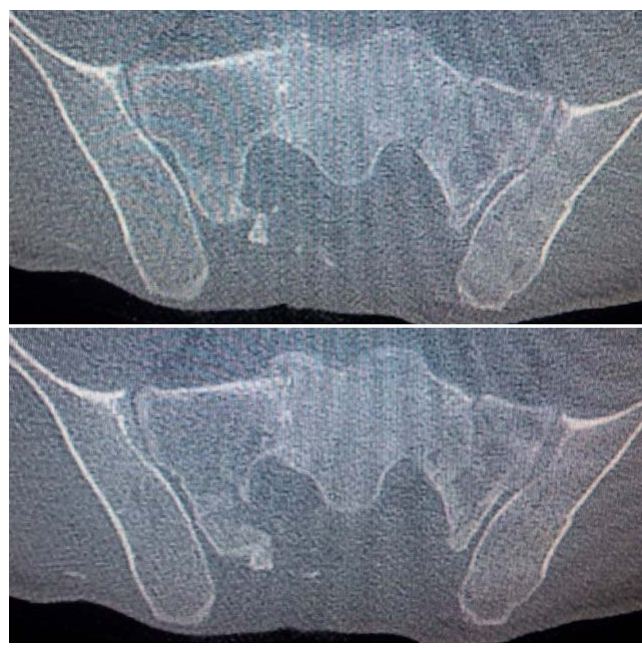

Figure 4. Three-month follow-up axial CT scan images showing sacroiliac bony fusion.

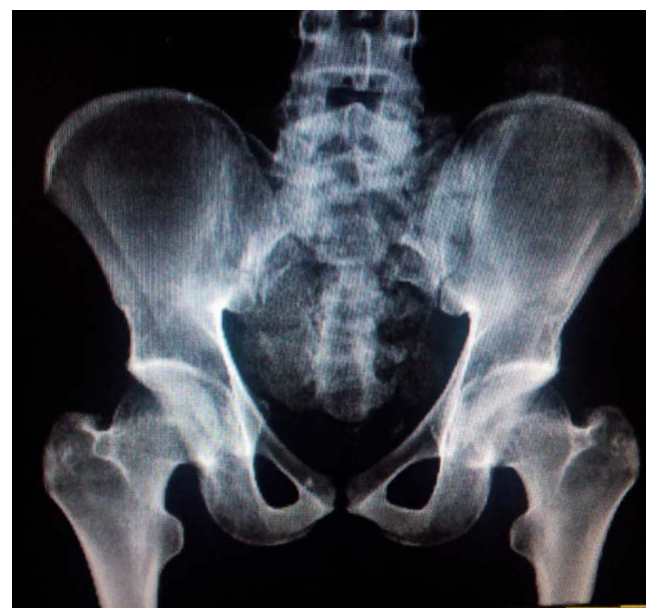

Figure 5. Three-month follow-up anteroposterior x-ray of the pelvis.

\section{Discussion}

Sacral fractures are usually seen together with pelvic ring fractures as a consequence of a blast injury, resulting in instability in nearly $30 \%$ of cases [3]. Multiplanar posterior pelvic ring instabilities are severe injuries, typically occurring in the sacroiliac joint, the os ilium, the sacrum or in a combination of these sites and their reconstruction is rather challenging, especially when they are associated with multiplanar fractures of the sacrum and the lumbosacral junction [4]. The mainstay of treatment is an aggressive stabilization and fixation that must be performed without any delay [3]. Lumbosacral and pelvic screw fixation is a well-documented technique for stabilizing fractured lumbosacral spine and pelvis [5]. Surgical stabilization of the lumbosacral spine and pelvis facilitates healing and earlier mobilization, but carries a high risk of postoperative infection [6]. On the other hand, conservative treatment avoids the undesirable sequelae of instrumentation infection and consequent need for hardware removal, but is associated with a rather prolonged hospital stay and a delayed patient ambulation.

\section{Conclusion}

This case report signifies that conservative treatment, with adjunctive therapies such as hyberbaric oxygen, may be an alternative option even for patients with complicated spinal injuries with axonal instability, when there are severe comorbidities or contraindications for surgical stabilization. Hyperbaric oxygen should be part of the armamentarium of every physician who treats patients with soft tissue infection and fractures.

\section{References}

1. Stuhmiller JH (2010) Blast Injury Translating Research Into Operational Medicine. In: William RS, Karl EF (Eds.), PhD, Colonel, US Army. Borden institute.

2. Rosenfeld JV, Bell RS, Armonda R (2015) Current concepts in penetrating and blast injury to the central nervous system. World J Surg 39: 1352-1362.[Crossref]

3. Dalbayrak S, Yaman O, Ayten M, Yilmaz M, Ozer AF (2014) Surgical treatment in sacral fractures and traumatic spinopelvic instabilities. Turk Neurosurg 24: 498-505. [Crossref]

4. Dudda M, Hoffmann M, Schildhauer TA (2013) [Sacrum fractures and lumbopelvic instabilities in pelvic ring injuries: classification and biomechanical aspects] Unfallchirurg 116: 972-978.[Crossref]

5. Liu G, Hasan MY, Wong HK (2016) Minimally invasive iliac screw fixation in treating painful metastatic lumbosacral deformity: a technique description and clinical results. Eur Spine J(Epub ahead of print) [Crossref]

6. Helgeson MD, Lehman RA Jr, Cooper P, Frisch M, Andersen RC, et al. (2011) Retrospective review of lumbosacral dissociations in blast injuries. Spine (Phila Pa 1976) 36: E469-475.[Crossref]

Copyright: (C2016 Spanos I. This is an open-access article distributed under the terms of the Creative Commons Attribution License, which permits unrestricted use, distribution, and reproduction in any medium, provided the original author and source are credited. 